\title{
Correction: Predictive modeling for odor character of a chemical using machine learning combined with natural language processing
}

Yuji Nozaki, Takamichi Nakamoto

There are errors in S2 Table. The values in the "Applicable descriptor Number (from S1 Table)" column are incorrect and should be 1 value lower. Additionally, the sensory dataset which was used in the computer simulation in S2 Table, is not perfectly equivalent to the original data source, Sigma-Aldrich's "Flavors and Fragrances" [2]. Therefore, S2 Table data are different from the original source. Some of the descriptors may be ignored for samples described by more than 6 descriptors. As descriptors are listed ascending in alphabet, ignored descriptors are mainly: "sweet", "vanilla" and "wine-like". Those descriptors are used when the number of descriptors is not more than six. Approximately $11 \%$ of samples in the dataset affected.

Please see the corrected S2 Table caption and file below.

\section{Supporting information}

S2 Table. Odor character profile of chemicals. S2 data set is different from original source. (CSV)

\section{Reference}

1. Nozaki Y, Nakamoto T (2018) Predictive modeling for odor character of a chemical using machine learning combined with natural language processing. PLoS ONE 13(6): e0198475. https://doi.org/10. 1371/journal.pone.0198475 PMID: 29902194

2. Sigma-Aldrich, "Flavors and Fragrances." [Online]. Available: http://go.sigmaaldrich.com/ff-catalogdownload-safcglobal.

\section{G OPEN ACCESS}

Citation: Nozaki Y, Nakamoto T (2018) Correction: Predictive modeling for odor character of a chemical using machine learning combined with natural language processing. PLOS ONE 13(12): e0208962. https://doi.org/10.1371/journal. pone.0208962

Published: December 5, 2018

Copyright: @ 2018 Nozaki, Nakamoto. This is an open access article distributed under the terms of the Creative Commons Attribution License, which permits unrestricted use, distribution, and reproduction in any medium, provided the original author and source are credited. 